\title{
SALUD
}

\section{Requerimiento calórico y su disponibilidad para estudiantes universitarios de la Carrera de Medicina y Cirugía de la Universidad Nacional de Asunción-Sajonia, 2018}

\author{
Anthon Torres-Romero', María Belén Torres, Divina Torres , María \\ Gabriela Troche1, Cecilia Ubaldi ${ }^{1}$, Miguel Valdés ${ }^{1}$, Ana Vargas ${ }^{1}$, Laura \\ Vargas ${ }^{1}$, Lucas Vázquez ${ }^{1}$, Arturo Vázquez ${ }^{1}$
}

\section{Resumen}

Introducción: Varios autores han destacado que la población universitaria es un grupo caracteriza por omitir comidas con frecuencia y realizar reiteradas ingestas entre las comidas principales, también su dieta se ve influenciada por una mayor independencia, la influencia de determinados hábitos sociales, su mayor disponibilidad de dinero que posibilita un gasto propio independiente, la oferta de restaurantes de comidas rápidas y la disponibilidad de alimentos precocinados para consumir en casa. Por lo que se trata de un periodo de educación crítico para el desarrollo de hábitos dietéticos que tienen mucha importancia en el estado de salud futuro. Los países de Latinoamérica, están experimentando un fenómeno que se conoce como transición nutricional, asociado al rápido proceso de urbanización y cambios económicos donde estas transiciones implican cambios en el patrón alimentario. Mostrándose que el tener conocimientos de nutrición, no influye en la toma de decisiones para una alimentación y estilo de vida saludables. La Federación de las Naciones Unidas para la Agricultura y la Alimentación (FAO) recomienda una ingesta calórica no mayor a 2000Kcal/día.

Objetivo: Determinar el requerimiento e ingesta calórica en estudiantes universitarios de la carrera de cirugía y medicina de la Universidad Nacional de Asunción-Sajonia, 2018.

Material y Método: Estudio observacional analítico, corte trasversal, no probabilístico por conveniencia. Abarcará desde 25 de septiembre hasta 18 de octubre del 2018. Se realizó en las instalaciones de la Facultad de Ciencias Médicas (sede Sajonia). Con una muestra constituida por un total de 202 encuestas, correspondientes a estudiantes del primer al tercer año de la carrera de Medicina y Cirugía de la UNA, Paraguay. Se aplicó la ecuación del Método FAOOMS para determinar el requerimiento calórico. Se utilizó la fórmula del Peso

1 Universidad Nacional de Asunción. Estudiantes de la Facultad de Ciencias Médicas en la carrera de Medicina y Cirugía.

E-mail: antiel.ts.ro@gmail.com

DOI: 10.26885/rcei.foro.2018.122 
dividido el cuadrado de la altura para determinar el Índice de Masa Corporal. Se siguieron los estándares de FAO para determinar las Kilocalorias ofrecidas en los comedores disponibles de la zona. La información se resume en tablas y gráficos, considerando una $\mathrm{p}<0,05$ como significativa en todos los casos.

Resultados: Se analizaron 202 casos; 117 de 1er curso, 41 de 2do curso, 44 de 3 er curso. Edades comprendidas entre 18 y 30 años $(21,04 \pm 1,56)$. El Gasto

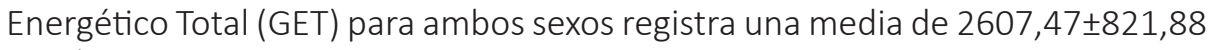
$\mathrm{Kcal} /$ día. La media de Kcal ofrecidas por los seis lugares de suministro aledaños a la facultad es de $2288,17 \pm 146,38 \mathrm{Kcal}$. Se encontró que los estudiantes realizan una media de 12,24 horas por día y una actividad moderada de 1,14 horas por día lo cual indica poca actividad. La mayoría de los estudiantes tienden al sedentarismo. Se encontró una correlación de Pearson significativa entre el IMC y el GET $(r=0,415)$. Al mismo tiempo, una correlación significativa entre Edad y IMC $(r=0,151)$.

Conclusiones: Los resultados muestran una alta necesidad calórica en los estudiantes de medicina, destacando la población masculina como mayormente afectada. La disponibilidad calórica excede al gasto calórico diario en la media mañana, almuerzo, merienda y cena. Se requiere un estudio detallado acerca de la relación entre el gasto energético total y la tendencia a la obesidad.

Palabras clave: requerimiento calórico, GET, comedores, universitarios, medicina.

\section{Referencias}

Pérez-Llamas, F., Garaulet, M., Herrero, F, et al. (2004). Una aplicación informática multivalente para estudios del estado nutricional de grupos de población. Valoración de la ingesta alimentaria. Nutrición Hospitalaria, 19(3), 160-166.

Pinheiro Volp, A. C., Esteves de Oliveira, F. C., Duarte Moreira Alves, R., Esteves E. A., Bressan J. (2011). Energy expenditure: components and evaluation methods. Nutr. Hosp. Jun, 26(3), 430-440. Recuperado de http://scielo.isciii.es/scielo.php?script=sci_arttext\&pid=S0212$16112011000300002 \& \operatorname{lng}=\mathrm{es}$

Troncoso, P. C., Amaya, P. J. P. (2009). Factores sociales en las conductas alimentarias de estudiantes universitarios. Revista Chilena de Nutrición, 36(4), 1090-1097. 